\title{
Fitz-Hugh-Curtis syndrome
}

\author{
Ch. P. Theofanakis $\cdot$ A. V. Kyriakidis
}

Received: 25 October 2010 / Accepted: 14 November 2010 / Published online: 7 December 2010

(C) Springer-Verlag 2010

\begin{abstract}
Fitz-Hugh-Curtis syndrome is characterized by perihepatic inflammation appearing with pelvic inflammatory disease (PID), mostly in women of childbearing age. Acute pain and tenderness in the right upper abdomen is the most common symptom that makes women visit the emergency rooms. It can also emerge with fever, nausea, vomiting, and, in fewer cases, pain in the left upper abdomen. It seems that the pathogens that are mostly responsible for this situation is Chlamydia trachomatis and Neisseria gonorrhoeae. Because of its characteristics, differential diagnosis for this syndrome is a constant, as it mimics many known diseases, such as cholelithiasis, cholecystitis, and pulmonary embolism. The development of CT scanning provided diagnosticians with a very useful tool in the process of recognizing and analyzing the syndrome. Nevertheless, the only secure diagnostic method is the laparoscopic evaluation of the abdomen and the spotting of the unique "violin-string" adhesions between the liver and the abdominal wall or the diaphragm. Though it is a difficult diagnosis, the syndrome and, therefore, the PID that caused its outburst are usually easily treated with courses of antibiotics, as monotherapy or combined, with satisfactory results.
\end{abstract}

Keywords Fitz-Hugh-Curtis syndrome $\cdot$ Pelvic inflammatory disease $\cdot$ Perihepatitis $\cdot$ Liver adhesions . Chlamydia trachomatis $\cdot$ Neisseria gonorrhoeae

C. P. Theofanakis · A. V. Kyriakidis

Department of General Surgery, General Hospital of Amfissa, Amfissa, Fokis, Greece

A. V. Kyriakidis $(\bowtie)$

Frouriou 95,

33100, Amfissa, Greece

e-mail: alkidi@hotmail.com

\section{Background}

The Fitz-Hugh-Curtis syndrome, perihepatitis associated with pelvic inflammatory disease (PID) [1], was first described by Carlos Stajano in 1920 to the Society of Obstetricians and Gynecologists of Montevideo in Uruguay [2]. Ten years later, in 1930, Thomas Fitz-Hugh and Arthur Curtis took the description of the syndrome one step further by connecting the acute clinical syndrome of right upper quadrant pain due to pelvic infection with the "violinstring" adhesions (Fig. 1) present in women with signs of prior salpingitis [3, 4]. After having studied several cases of patients with gonococcal disease, baring these adhesions between the liver and the abdominal wall, Curtis demonstrated a couple of years later that these signs are absent in other cases of peritonitis, thus making this entity a unique syndrome [5]. Also, Fitz-Hugh demonstrated that $N$. gonorrhoeae should be the pathogenetic cause of the syndrome, after having isolated gram-negative diplococci on liver capsule smears [4].

For a great amount of time, $N$. gonorrhoeae was thought to be the sole pathogenetic cause of the syndrome. However, in 1978, Muller-Schoop et al. presented, for the first time, evidence that patients who had undergone laparoscopic evaluation due to peritonitis were also diagnosed with perihepatitis and were infected with Chlamydia trachomatis [6]. Paavonen et al. and Wang et al. came to the same conclusion in their own studies a few years later $[7,8]$. This pathogen has also been isolated from the cervix, the fallopian tubes, and, in some cases, the liver capsule in patients with perihepatitis, thus making $C$. trachomatis a more common pathogen than $N$. gonorrhoeae in patients with Fitz-Hugh-Curtis syndrome [9, 10].

Fitz-Hugh-Curtis syndrome is more common in women of reproductive age who visit hospitals due to acute pain and 


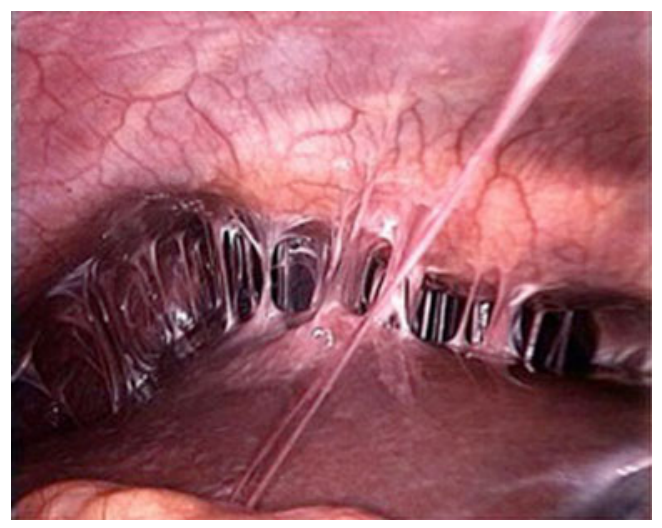

Fig. 1 "Violin-string" adhesions of chronic Fitz-Hugh-Curtis syndrome [31]

tenderness in the right upper abdomen [11]. This pain is caused by adhesion of the anterior hepatic surface and the abdominal wall [12]. Differential diagnosis from acute cholecystitis, pleurisy, and right pyelonephritis is difficult because the pain becomes more severe upon body movement and breathing. In some cases, the pain in the right upper abdomen is absent, and there is pain only in the lower abdomen, mimicking acute appendicitis or peritonitis $[13,14]$.

\section{Methods and findings}

\section{Incidence of Fitz-Hugh-Curtis syndrome}

It has been well established that the frequency of the diagnosis of Fitz-Hugh-Curtis syndrome depends on the diagnostic criteria used. It is possible for patients with no symptoms to have extended perihepatic adhesions recognized through laparoscopy, while others with PID and right upper quadrant pain might have no laparoscopic signs or any kind of evidence of perihepatitis [1].

In a study published in 1979, Semchyshyn et al. found perihepatitis in $12 \%$ of patients having PID, using only clinical criteria [15]. Onsrud et al. added laparoscopic criteria and found a $13.8 \%$ rate of perihepatitis combined with PID [16]. This 2-year study included all patients with PID who were examined in one hospital, from which $37 \%$ had no right upper quadrant pain but had strong laparoscopic evidence of both PID and perihepatitis. Women who had undergone placement of an intrauterine contraceptive device (IUD) within the previous 6 weeks had higher laparoscopic evidence of perihepatitis than those who retained such device for a longer period. Other studies from Paavonen et al. and Wang et al. demonstrated significantly lower laparoscopic and clinical evidence of perihepatitis related to PID $[7,8]$.

However, a study conducted in the adolescent population by Litt and Cohen showed that clinical evidence of perihepa- titis was found in $27 \%$ of patients with salpingitis. This higher rate could be explained as due to anatomical differences in the genital tract between adolescents and adults that could enable progression of cervicitis to PID and, probably, to Fitz-HughCurtis syndrome. It is known that the transitional zone between the squamous and columnar epithelium of the cervix (ectropion) is at the outer margin of the cervix in adolescents, while within the cervix in adults [17].

Gatt et al. in 1986 presented seven cases of Fitz-HughCurtis syndrome diagnosed in a period of 6 months. All patients were diagnosed as acute surgical emergencies, but only one had right upper quadrant pain as the presenting symptom. Nevertheless, pain was present in all cases to a variable extent. Differential diagnosis was made from acute appendicitis, pulmonary embolism, left renal colic, acute cholecystitis, chronic cholecystitis, and urinary tract infection. In five cases, symptoms were initiated with a complicated termination of pregnancy, and in one case, a hysterectomy seemed to be the cause of PID. Diagnosis of Fitz-Hugh-Curtis syndrome was established through laparoscopy, which demonstrated signs of perihepatitis in association with PID. Moreover, six out of seven patients had microbiological or serological evidence of chlamydial infection, and all patients responded satisfactorily to a 4-week course of oral tetracycline [18].

Woo et al., trying to analyze the clinical characteristics of Fitz-Hugh-Curtis syndrome, conducted a study from January 2005 to December 2006, and 22 female patients of childbearing age were diagnosed with the syndrome. The diagnostic standard was abdominal CT scan that showed pelvic inflammation with contrast enhancement of hepatic capsules and adhesions between the liver and the diaphragm or the liver and the abdominal wall detected by laparoscopy. Twenty out of 22 patients visited the emergency room due to acute abdominal pain, $32 \%$ of which was spotted in the right upper abdomen, $32 \%$ both in the right upper abdomen and in the lower abdomen, $27 \%$ only in the lower abdomen, while $4.5 \%$ presented with fever and $4.5 \%$ with epigastric pain [11].

\section{Pathogenesis of Fitz-Hugh-Curtis syndrome}

Pathogenesis of the syndrome is still a puzzle for clinicians because the mechanism that leads to Fitz-Hugh-Curtis syndrome is not fully understood. Many theories have been proposed over the years, and research is still ongoing.

In 1953, Holm-Nielsen et al. suggested that pathogenic organisms spread through the peritoneal fluid from the pelvis to the diaphragm, especially if there has been recent use of an IUD [16, 19]. On the other hand, the isolation of bacteria from the surface of the liver or the surrounding ascites is not that common in patients with Fitz-HughCurtis syndrome [10]. 
A different theory was suggested through a case report by Banerjee et al., in which the spread of the infection could take place via the blood stream. The hematogenous spread was supported due to the finding of focal lesions in a patient with Fitz-Hugh-Curtis syndrome, which resolved with the use of antibiotics [20].

Another theory is the spread through the lymphatic tract, and this could explain the fact that most patients have no signs of an extensive intra-abdominal infection or disseminated blood stream infection. However, Lopez-Zeno et al. demonstrated that the female reproductive tract is retroperitoneal, thus, there is no direct connection between the pelvic and the subdiaphragmatic lymph nodes [12].

During the past decades, researchers have formulated theories according to which the outburst of Fitz-Hugh-Curtis syndrome is part of a hyperimmune response to C. trachomatis. Studies demonstrated that patients with perihepatitis and salpingitis had higher titers of IgG antibodies than the ones that were diagnosed with perihepatitis alone. In a paper published in 1997, Money et al. compared 27 patients with perihepatitis and salpingitis, diagnosed via laparoscopy, and 46 patients with salpingitis alone. According to their results, $67 \%$ of the patients in the perihepatitis-salpingitis group was found with elevated levels of antibodies to Chsp60, in contrast to $28 \%$ of the patients of the salpingitis-alone group. In addition, antichlamydial antibodies $\operatorname{IgG}$ and IgM were not statistically different between the two groups, and the authors concluded that the Chsp60 antigen has an influence in the inflammatory response of each patient [21].

\section{Signs and symptoms}

The most common symptom that patients with Fitz-HughCurtis syndrome who visit the emergency rooms experience is usually acute abdominal pain. The pain in the right upper abdomen is usually associated with biliary disease like gall bladder stones or cholecystitis, but clinicians have to consider also potential duodenal ulcers, liver abscess, subphrenic abscess, and herpes zoster infection. The diagnostic problem emerges when right upper abdominal pain is combined with lower abdominal pain. This situation is mostly associated with Fitz-Hugh-Curtis syndrome, although sometimes, the pain in the right upper abdomen can be the only sign of the disease. In such cases, the pain is mimicking acute gall bladder disease, posing a diagnostic challenge.

The syndrome usually emerges with fever, abdominal pain, and vaginal discharge, due to PID [22]. The inflammation of the upper side of the diaphragm usually causes a sharp right upper quadrant pain; pain can also be spotted at the right shoulder or the inside of the right arm, accompanied by nausea, vomiting, night sweats, headache, and malaise. Additionally, pain usually becomes stronger upon movement $[4,12]$. A pleuritic right-sided chest pain could also occur, mimicking pleurisy, pneumonia, and pulmonary embolism [23]. Nevertheless, there have been reports in the literature about Fitz-Hugh-Curtis syndrome patients complaining about left upper quadrant pain posing as perisplenitis, thus, with no participation of the liver [24].

\section{A diagnostic challenge}

As it was mentioned before, Fitz-Hugh-Curtis syndrome poses a diagnostic puzzle, as it mimics many known pathologic tracks (Table 1). Though it is usually set off by PID, it can be portrayed as left renal colic, urinary tract infection, acute appendicitis, pulmonary embolism, and acute or chronic cholecystitis [18].

Studies have shown that the only secure way to diagnose a patient with Fitz-Hugh-Curtis syndrome is a laparoscopic exploration of the abdomen. Laparoscopy can also be used in order to solve the characteristic "violin-string" adhesions. However, this is not the first choice since the goal is to recognize first the underlying symptoms of PID. Physical examination reveals severe tenderness in the right upper quadrant, while a friction rub that could be heard along the right anterior costal margin was described by Fitz-Hugh as "beautiful new snow creaking frictions" [4]. Vaginal discharge, cervical motion tenderness, and adnexal tenderness, diagnosed via a bimanual pelvic examination, are also signs of PID [1].

Ultrasonography is used in these cases in order to evaluate the gallbladder and the liver, thus excluding cholelithiasis and cholecystitis, while a thorough look at the ovaries for abscesses could indicate PID.

Wang et al. demonstrated that Fitz-Hugh-Curtis syndrome could be diagnosed radiologically. A CT scan could show the thickening of the hepatic capsule and the collection of

Table 1 Differential diagnosis of Fitz-Hugh-Curtis syndrome [1]

Differential diagnosis of Fitz-Hugh-Curtis syndrome

\begin{tabular}{ll}
\hline Cholelithiasis & Subphrenic abscess \\
Cholecystitis & Pancreatitis \\
Pleurisy & Appendicitis \\
Pneumonia & Herpes zoster \\
Pulmonary embolism & Enteroviral epidemic pleurodynia \\
& (Bornholm disease)
\end{tabular}

Rib fracture

Pyelonephritis

Hepatitis

Nephrolithiasis

Perforated ulcer 
subcapsular fluid, while enhanced CT could strikingly reveal thickening of the hepatic capsule and its uniform enhancement in the arterial phase and patching or wedging enhancement of the involved liver parenchyma [25]. Sam et al. also managed to demonstrate sufficient data for the diagnosis of the syndrome through contrast-enhanced CT scans [32; Fig. 2].

Treating PID to cure Fitz-Hugh-Curtis syndrome

Since Fitz-Hugh-Curtis syndrome emerges from PID, symptomatic therapy is the key. Judlin et al. published recent data from the MONALISA study, according to which a fourth-generation synthetic fluoroquinolone antibacterial agent, moxifloxacin, is an ideal monotherapy for the treatment of PID [27]. Nevertheless, antibiotic therapy should be directed to the most likely pathogens, such as $N$. gonorrhoeae and C. trachomatis and microorganisms found in the endogenous flora of the vagina and cervix, such as anaerobic bacteria and facultative bacteria, many of which are associated with bacterial vaginosis $[28,29]$.

The Centers for Disease Control and Prevention are undergoing the 2010 guidelines for the treatment of STDs. However, the 2006 guidelines, with a 2007 update for PID, as described on Tables 2 and 3, is the safest path for clinicians worldwide. Parenteral and oral therapies appear to have similar clinical efficacy in treating women with PID of mild or moderate severity. The gold standard seems to be cefotetan 2 g IV every $12 \mathrm{~h}$ or cefoxitin 2 g IV every $6 \mathrm{~h}$ with doxycycline $100 \mathrm{mg}$ orally or IV every $12 \mathrm{~h}$ for parenteral treatment and ceftriaxone $250 \mathrm{mg}$ IM in a single dose with doxycycline $100 \mathrm{mg}$ orally twice a day for 14 days, with or without metronidazole $500 \mathrm{mg}$ orally twice a day for 14 days, for per os treatment. Clinical experience should guide decisions regarding transition to oral therapy,

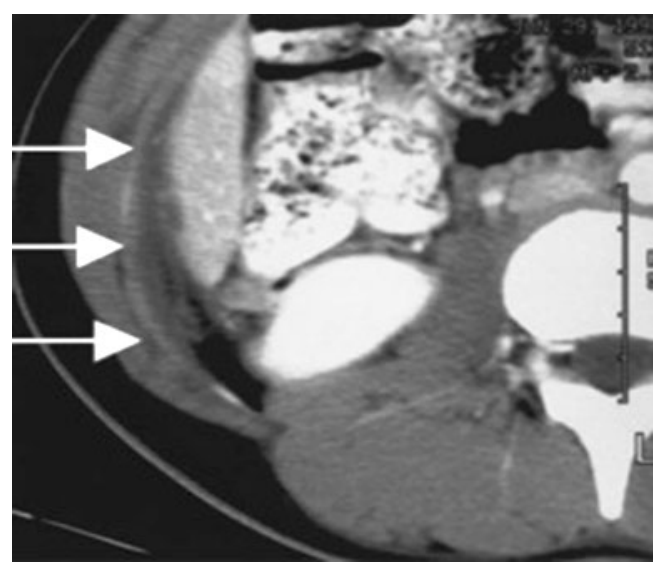

Fig. 2 Fitz-Hugh-Curtis syndrome: contrast-enhanced CT scans of the right upper abdominal quadrant demonstrate inflammatory stranding along the right paracolic gutter and inferior right lobe of the liver [26]
Table 2 Recommended parenteral regimens [30]

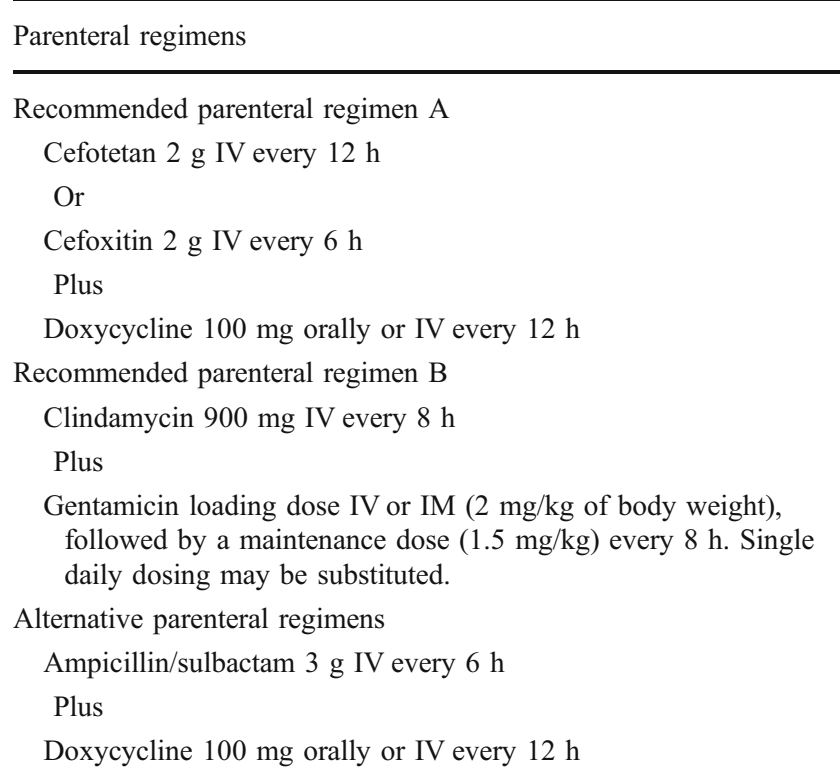

which usually can be initiated within $24 \mathrm{~h}$ of clinical improvement. Oral therapy can be considered for women with mild to moderately severe acute PID, as the clinical outcomes among women treated with oral therapy are similar to those treated with parenteral therapy. Women who do not respond to oral therapy within $72 \mathrm{~h}$ should be reevaluated to confirm the diagnosis and should be administered parenteral therapy on either an outpatient or inpatient basis.

Table 3 Recommended oral regimens [30]

Recommended oral regimen

Ceftriaxone $250 \mathrm{mg}$ IM in a single dose

Plus

Doxycycline $100 \mathrm{mg}$ orally twice a day for 14 days

With or without

Metronidazole $500 \mathrm{mg}$ orally twice a day for 14 days

Or

Cefoxitin $2 \mathrm{~g}$ IM in a single dose and probenecid $1 \mathrm{~g}$ orally administered concurrently in a single dose

Plus

Doxycycline $100 \mathrm{mg}$ orally twice a day for 14 days

With or without

Metronidazole $500 \mathrm{mg}$ orally twice a day for 14 days

Or

Other parenteral third-generation cephalosporins

(e.g., ceftizoxime or cefotaxime)

Plus

Doxycycline $100 \mathrm{mg}$ orally twice a day for 14 days

With or without

Metronidazole $500 \mathrm{mg}$ orally twice a day for 14 days 
Laparoscopy should be used, as it is less invasive than laparotomy, in order for patients to cope with the complications of PID, such as adhesions, mostly in the perihepatic area, the lysis of which leads to symptom relief $[1,29]$.

If parenteral cephalosporin therapy is not feasible, use of fluoroquinolones (levofloxacin $500 \mathrm{mg}$ orally once daily or ofloxacin $400 \mathrm{mg}$ twice daily for 14 days) with or without metronidazole (500 mg orally twice daily for 14 days) may be considered if the community prevalence and individual risk of gonorrhea are low. Tests for gonorrhea must be performed prior to instituting therapy, and the patient must be managed as follows if the test is positive:

If nucleic acid amplification test is positive, parenteral administration of cephalosporin is recommended.

If culture for gonorrhea is positive, treatment should be based on results of antimicrobial susceptibility. If the isolate is quinolone-resistant $N$. gonorrhoeae or if antimicrobial susceptibility cannot be assessed, parenteral administration of cephalosporin is recommended.

Although information regarding other outpatient regimens is limited, amoxicillin/clavulanic acid and doxycycline or azithromycin with metronidazole have demonstrated a shortterm clinical cure. No data have been published regarding the use of oral cephalosporins for the treatment of PID [30].

\section{Conclusions}

Fitz-Hugh-Curtis syndrome is a common clinical entity connected with perihepatitis and PID that, however, needs to be treated with great attention. It seems that $C$. trachomatis and $N$. gonorrhoeae are implicated in the pathogenesis of the syndrome. Differential diagnosis is often a challenging procedure since its symptoms mimic severe surgical entities, such as pancreatitis, appendicitis, perforated ulcer, cholecystitis, and more. Abdominal CT scans can reveal the subject PID, but the syndrome can be safely diagnosed via laparoscopy. The development of new technologies in this minimally invasive technique can help a great number of patients to avoid unnecessary surgery, unpleasant pain, and lingering suffering. Early treatment with antibiotic therapy, combined with lysis of the adhesions through laparoscopy, can lead to the treatment of PID responsible for the outburst of the syndrome. Nevertheless, it is important to point out that abdominal infections, especially those affecting the genital tract, should be diagnosed and treated in their early stages since the risk of permanent damages and pelvic sepsis is not to be taken lightly.

Declaration of interest The authors report no conflicts of interest. The authors alone are responsible for the content and writing of the paper.

\section{References}

1. Peter NG, Clark LR, Jaeger JR (2004) Fitz-Hugh-Curtis syndrome: a diagnosis to consider in women with right upper quadrant pain. Cleve Clin J Med 71(3):233-239

2. Stajano C (1920) La reaccion frenica en ginecologia. Sem Med 27:243-248

3. Curtis A (1930) A cause of adhesions in the right upper quadrant. JAMA 94:1221-1222

4. Fitz-Hugh TJ (1934) Acute gonococcic peritonitis of the right upper quadrant in women. JAMA 102:2094-2096

5. Curtis A (1932) Adhesions of the anterior surface of the liver. JAMA 99:2010-2012

6. Muller-Schoop JW, Wang SP, Munzinger J, Schlapfer HU, Knoblauch M, Tammann RW (1978) Chlamydia trachomatis as possible cause of peritonitis and perihepatitis in young women. $\mathrm{Br}$ Med J 1:1022-1024

7. Paavonen J, Saikku P, von Knorring J, Aho K, Wang SP (1981) Association of infection with Chlamydia trachomatis with FitzHugh-Curtis syndrome. J Infect Dis 144(2):176

8. Wang SP, Eschenbach DA, Holmes KK, Wager G, Grayston JT (1980) Chlamydia trachomatis infection in Fitz-Hugh-Curtis syndrome. Am J Obstet Gynecol 138(7 Pt 2):1034-1038

9. Dalaker K, Gjonnaess H, Kvile G, Urnes A, Anestad G, Bergan T (1981) Chlamydia trachomatis as a cause of acute perihepatitis associated with pelvic inflammatory disease. $\mathrm{Br} \mathrm{J}$ Vener Dis $57: 41-43$

10. Wolner-Hanssen P, Svensson L, Westrom L, Mardh PA (1982) Chlamydia trachomatis from the liver capsule in Fitz-HughCurtis syndrome. N Engl J Med 306:113

11. Woo SY, Kim JL, Cheung DY, Cho SH, Park SH, Han JY et al (2008) Clinical outcome of Fitz-Hugh-Curtis syndrome mimicking acute biliary disease. World J Gastroenterol 14(45):6975-6980

12. Lopez-Zeno JA, Keith LG, Berger GS (1985) The Fitz-HughCurtis syndrome revisited. Changing perspectives after half a century. J Reprod Med 30:567-582

13. Shanahan D, Gau D (1986) Chlamydial Fitz-Hugh/Curtis syndrome. Lancet 1(8491):1216

14. Wood JJ, Bolton JP, Cannon SR, Allan A, O'Connor BH, Darougar S (1982) Biliary-type pain as a manifestation of genital tract infection: the Curtis-Fitz-Hugh syndrome. Br J Surg 69:251253

15. Semchyshyn S (1979) Fitz-Hugh and Curtis syndrome. J Reprod Med 22(1):45-48

16. Onsrud M (1979) Perihepatitis in salpingitis. Fitz-Hugh-Curtis syndrome. Tidsskr Nor Laegeforen 99(33):1705-1706

17. Litt IF, Cohen MI (1978) Perihepatitis associated with salpingitis in adolescents. JAMA 240(12):1253-1254

18. Gatt D, Heafield T, Jantet G (1986) Curtis-Fitz-Hugh syndrome: the new mimicking disease? Ann R Coll Surg Engl 68(5):271-274

19. Holm-Nielsen P (1953) Right upper quadrant pain in salpingitis and other abdominal diseases explained by adsorption of exudates from the peritoneal cavity through the diaphragm. Acta Chir Scan 104:435-446

20. Banerjee B, Rennison A, Boyes BE (1992) Sonographic features in a case of Fitz-Hugh-Curtis syndrome masquerading as malignancy. Br J Radiol 65:342-344

21. Money DM, Hawes SE, Eschenbach DA, Peeling RW, Brunham R, Wolner-Hanssen P et al (1997) Antibodies to the chlamydial 60 $\mathrm{kd}$ heat-shock protein are associated with laparoscopically confirmed perihepatitis. Am J Obstet Gynecol 176(4):870-877

22. Ris HW (1984) Perihepatitis (Fitz-Hugh-Curtis syndrome). A review and case presentation. J Adolesc Health Care 5:272-276

23. Bolton JP, Darougar S (1983) Perihepatitis. Br Med Bull 39:159162 
24. Gatt D, Jantet G (1987) Perisplenitis and perinephritis in the Curtis-Fitz-Hugh syndrome. Br J Surg 74:110-112

25. Wang C, Guo X, Yuan Z, Shi Q, Hu X, Fang L (2009) Radiologic diagnosis of Fitz-Hugh-Curtis syndrome. Chin Med J 122(6):741-744

26. Judlin P, Liao Q, Liu Z, Reimnitz P, Hampel B, Arvis P (2010) Efficacy and safety of moxifloxacin in uncomplicated pelvic inflammatory disease: the MONALISA study. BJOG 117:1475-1484

27. McCormack WM (1994) Pelvic inflammatory disease. N Engl J Med 330(2):115-119

28. Sweet RL (2009) Treatment strategies for pelvic inflammatory disease. Expert Opin Pharmacother 10:823-837
29. Reichert JA (1976) Fitz-Hugh-Curtis syndrome. A laparoscopic approach. JAMA 236:266-268

30. CDC guidelines for treating pelvic inflammatory disease. Available at: http://www.cdc.gov/std/treatment/2006/updated-regimens.htm. Accessed 18 Oct 2010

31. Moore-Shepherd S (2010) Pelvic inflammatory disease. Available at http://emedicine.medscape.com/article/256448-overview. Accessed 20 Oct 2010

32. Sam JW, Jacobs JE, Birnbaum BA (2002) Spectrum of CT findings in acute pyogenic pelvic inflammatory disease. Radiographics 22:1327-1334 